\title{
New Route from Si-O to Si-C bonds: Alkylalkoxysilanes from Alkoxysilanes and Metal Hydrides
}

Florian J. Schattenmann, * Woodfin V. Ligon, Paul Donahue, Hans Grade, Kara Abatto

GE Global Research Center, Niskayuna, NY 12309

Supplemental Information 


\section{General Methods}

All reactions were performed in a fixed-bed reactor setup as schematically represented in Supplemental Figure 1. Sodium hydride (95\% purity) was purchased from Aldrich and exclusively handled under an inert gas atmosphere. $\mathrm{Si}(\mathrm{OMe})_{4}(99.5 \%$ purity) was purchased from Aldrich and used as is. Analytical methods were performed as follows:

GC. The fractions collected at the reactor downstream were analyzed using a Hewlett Packard 6890 gas chromatograph with auto sampler. The GC setup was calibrated using known mixtures of tetramethoxysilane, methyltrimethoxysilane and trimethoxysilane and was periodically checked for repeatability.

GC/MS. Two microliters of the sample were drawn up into a $10 \mu \mathrm{l}$ syringe with a pocket of air in the syringe barrel above the aliquot of sample. Just before injection the $2 \mu \mathrm{l}$ sample was expelled leaving a "wet needle" which was immediately injected into the GC/MS. The injection split was approximately $10: 1$. The injector was operated at $300^{\circ} \mathrm{C}$ and the column was programmed from $50^{\circ} \mathrm{C}$ to $250^{\circ} \mathrm{C}$ at $10^{\circ} \mathrm{C} / \mathrm{min}$. The column was a $\mathrm{J} \& \mathrm{~W}$ Scientific model DB-5 MS with a length of 30 meters and a width of $0.52 \mathrm{~mm}$. The column had a film thickness of 1.5 micrometers. The carrier gas was hydrogen.

The mass spectrometer was a JEOL model HX110 double focusing magnetic mass spectrometer operated in electron ionization mode at 1000 resolution. The instrument was scanned from 10-800 amu at 1 second per full cycle (scan plus fly-back). Spectra obtained were compared with the National Institutes of Science and Technology (NIST) library.

NMR. All data were obtained on GE NMR Instruments Omega nuclear magnetic resonance spectrometers operating at $500 \mathrm{MHz}(11.75 \mathrm{~T})$ for ${ }^{13} \mathrm{C},{ }^{29} \mathrm{Si}$, and ${ }^{2} \mathrm{H}$ and at $300 \mathrm{MHz}(7.06 \mathrm{~T})$ for ${ }^{1} \mathrm{H}$. ${ }^{13} \mathrm{C}$, ${ }^{29} \mathrm{Si}$, and ${ }^{2} \mathrm{H}$ data were obtained using a $10 \mathrm{~mm}$ broadband probe and ${ }^{1} \mathrm{H}$ data was obtained using a 5 $\mathrm{mm}{ }^{1} \mathrm{H}-{ }^{13} \mathrm{C}$ dual frequency probe. All spectra were obtained using $\mathrm{CDCl}_{3}$ as the solvent. Acquisition 
parameters for ${ }^{13} \mathrm{C}$ (at $125.75 \mathrm{MHz}$ ) were a sweep width of $30.3 \mathrm{kHz}$ with a $11.2 \mathrm{kHz}$ offset and $32 \mathrm{k}$ data points resulting in a $1.08 \mathrm{~s}$ acquisition time. A one-pulse sequence was used with a $45^{\circ}$ flip and 1s pulse delay. A total of 5600 transients were accumulated. ${ }^{29} \mathrm{Si}$ (at $99.35 \mathrm{MHz}$ ) data was obtained using a $20.0 \mathrm{kHz}$ sweep width with a $-5.4 \mathrm{kHz}$ offset and 16k data points resulting in an $800 \mathrm{~ms}$ acquisition time. An inverse gated pulse sequence was used with broadband decoupling on during acquisition only with a $5 \mathrm{~s}$ pulse delay and $90^{\circ}$ flip angle. Chromium tris-acetyl acetonate was added to the solution as a spin-lattice relaxation reagent to reduce NOE. 9600 transients were accumulated. For ${ }^{2} \mathrm{H}$ (at $76.7 \mathrm{MHz}$ ) observe a $2.0 \mathrm{kHz}$ sweep width and $384 \mathrm{~Hz}$ offset were employed with $2 \mathrm{k}$ data points to give a $1.024 \mathrm{~s}$ acquisition time. The lock channel was turned off and the lock coil detuned to allow deuterium observation. An inverse gated pulse sequence was employed and 512 transients were obtained. ${ }^{1} \mathrm{H}$ NMR data was obtained at $300.1 \mathrm{MHz}$ using a simple one-pulse pulse sequence. A sweep width of $4.5 \mathrm{KHz}$ and $8 \mathrm{k}$ data points resulted in an acquisition time of $1.8 \mathrm{~s}$. A pulse delay of $8.2 \mathrm{~s}$ and a $30^{\circ}$ flip were also used to acquire 128 scans. All data was processed using an in-house data processing package NetNMR. A polynomial baseline correction was applied and a correction to compensate for Bessell filter drop off was also applied.

MS analysis of gas phase. A JEOL SX-102 mass spectrometer was used with the resolution set to 1000 and tuned to $\mathrm{m} / \mathrm{z}=2$. $250 \mu \mathrm{l}$ of gas phase was injected into an alumina Plot column (program: 3 min at $-80^{\circ} \mathrm{C}$; temperature ramp: $60^{\circ} \mathrm{C} / \mathrm{min}$ to $10^{\circ} \mathrm{C}$ followed by $25^{\circ} \mathrm{C} / \mathrm{min}$ up to $200^{\circ} \mathrm{C}$.

Elemental analysis of bed. The bed samples were ashed and fused in the presence of $\mathrm{Li}_{2} \mathrm{~B}_{4} \mathrm{O}_{7}$ in $\mathrm{Pt}$ crucibles. Then the samples were dissolved in $\mathrm{HNO}_{3}$ and diluted. The solutions were analyzed on a Varian Liberty II ICP-AES instrument. 
Fixed-bed reactor setup.

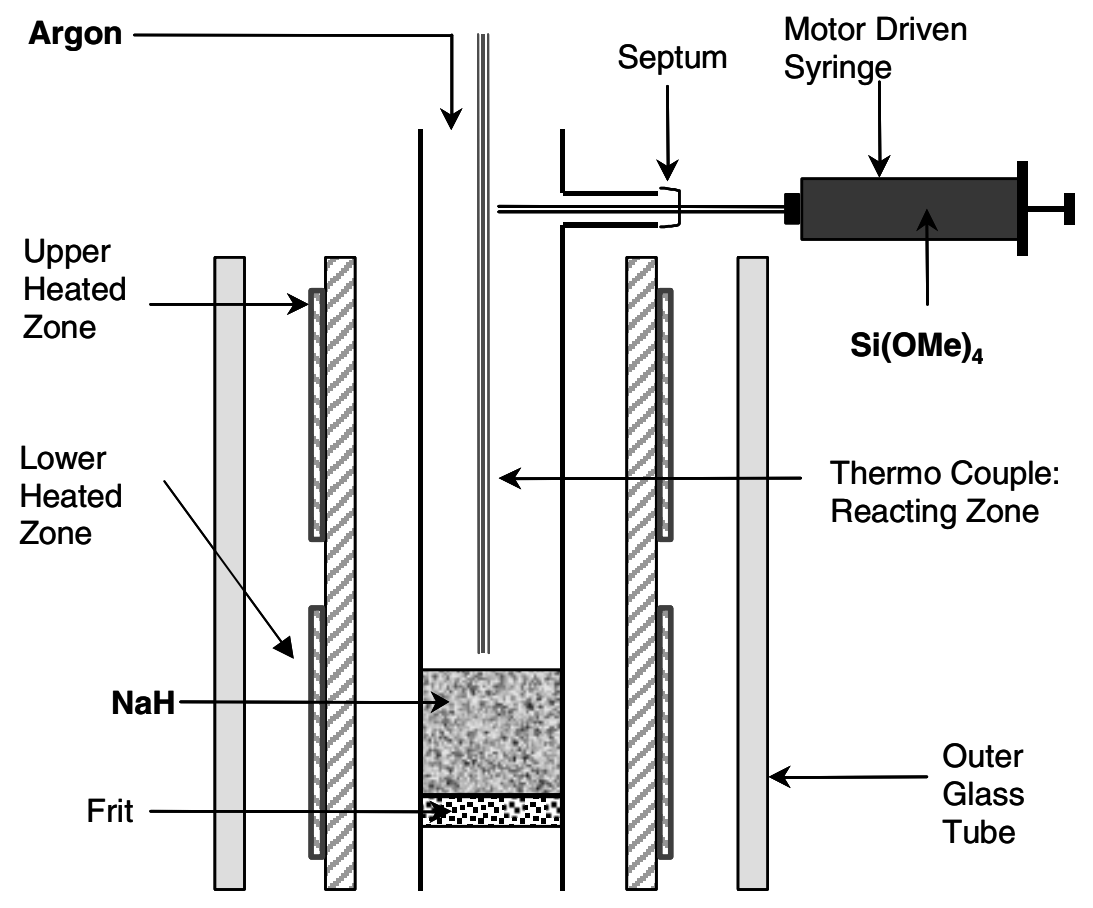

Supplemental Figure 1. Schematic representation of the fixed-bed reactor setup used in the reaction of tetramethoxysilane and $\mathrm{NaH}$. 